\title{
Cloacoscopy in the Horned Viper (Vipera ammodytes)
}

\author{
Matteo Oliveri ${ }^{1}$, Manuel Morici $^{2}$, Robert Novotný ${ }^{1}$, Alena Bartošková ${ }^{1}, Z_{\text {deněk Knotek }}^{1}$ \\ ${ }^{1}$ University of Veterinary and Pharmaceutical Sciences Brno, Faculty of Veterinary Medicine, \\ Avian and Exotic Animal Clinic, Brno, Czech Republic \\ ${ }^{2}$ University of Messina, Department of Veterinary Science, Veterinary Teaching Hospital, Messina, Italy
}

Received September 30, 2015

Accepted August 31, 2016

\begin{abstract}
This study illustrates the main morphological structures of the cloaca of six adult horned vipers Vipera ammodytes (3.3). The coprodeum is characterized by smooth and thin mucosa, with moderate vasculature. In the centre of the coprodeum the anal sphincter is located, which separates the coprodeum from the colon. Structures of the urodeum are divided by two highly developed septa in female vipers. The urodeum in female vipers is characterized by distinct chambers, which become visible immediately behind the exit of the sphincter of the coprodeum. Vaginal pouches leading to the entrance of the oviduct are visible. The male urodeum is characterized by two distinct structures. In the upper part of the urodeum, the coprodeum sphincter surrounded by a gently folded mucosa, and the urethral papillae surface on a large mucosal protrusion emerging from the dorsal urodeum are visible in male horned vipers. The mucosal protrusion that hosts the urethral papillae can be found in two different appearances, closed or open. The proctodeum is a tubular and muscular structure that leads to the vent. Cloacoscopy is as a safe and effective method for cloaca exploration in both sexes of the horned viper. This technique may be used to assist artificial insemination in the horned viper in the future.
\end{abstract}

Cloaca, endoscopy, urodeum, vaginal pouch, sex determination

Detailed knowledge of the snake cloacal anatomy is necessary for clinical evaluation, sex determination, and for artificial insemination programmes in endangered snake species. Literature information regarding the clinical anatomy of the snake cloaca is still scarce (Stahl 2006). The cloaca is not easily thoroughly evaluated during the physical examination of snakes. Rigid endoscopy is a safe and effective method to describe the majority of the most important anatomical structures in various reptiles (Jekl et al. 2007; Divers 2014; Di Girolamo and Selleri 2015; Knotek and Jek1 2015). The objective of this study was to describe the main morphological structures of the cloaca with a special regard to the urodeum and the structures annexed to the reproductive system in the horned viper (Vipera ammodytes) using the rigid endoscope.

\section{Materials and Methods}

Six adult horned vipers (3.3), were included in the study. All snakes originated from a private collection and were healthy. The weight of the animals ranged from 200 to $300 \mathrm{~g}$. All vipers were explored during the putative mating season between April and June 2015 (after a period of hibernation from November 2014 till March 2015). Cloacoscopy could be performed without any sedation or anaesthesia. Snakes were restrained using a plastic tube (Contention tubes, Midwest Tongs, USA), by gently forcing the head and the first third of the animal body inside the tube. Once the snake was safely placed in the tube, the animal was kept in dorsal recumbency. Cloacoscopy was performed with an ureteroscope ( $1 \mathrm{~mm}$ diameter, $45 \mathrm{~cm}$ length, $30^{\circ}$ angle, Karl Storz endoscope, Tuttlingen, Germany) including an endoscope sheath ( $2 \mathrm{~mm}$ diameter). Pictures were taken with an endo-camera (Veterinary Videocamera III, Karl Storz endoscope, Tuttlingen, Germany) and processed by special software (Debut Video Capture Software Professional v 1.64, NCH software, USA).

The ureteroscope was gently introduced into the cloaca through the vent and pushed 2 to $3 \mathrm{~cm}$ into the coprodeum. Sterile saline $(0.9 \% \mathrm{NaCl}, \mathrm{B} \backslash \mathrm{Braun}$, Germany) was flushed into the cloaca through the endoscope

Address for correspondence:

Dr. Matteo Oliveri

Avian and Exotic Animal Clinic

Faculty of Veterinary Medicine

University of Veterinary and Pharmaceutical Sciences Brno

Palackého tř.1946/1, 61242 Brno, Czech Republic 
sheath to clean the optic and dilate the cloacal mucosa. The saline was flushed at room temperature. Due to its minimal volume, the fresh saline used did not cause any discomfort to the vipers. The ureteroscope was then slowly withdrawn to allow visualization of the main structures of the cloaca, from the coprodeum to the urodeum and proctodeum. Once the urodeum was reached in the female viper, the optic was aligned with the entrance of the oviduct and a 4 Fr. cannula (Transcervical Insemination Catheter, Minitube, Germany) was gently inserted into the oviduct, overcoming the vaginal sphincter. Sex determination was confirmed by probing of the hemipenal pockets of the snakes following cloacoscopy.

\section{Results}

Once the optic is inserted, the coprodeum is readily visible. This structure is characterized by a smooth and thin mucosa, with moderate vasculature and is normally free from urates (Plate V, Fig. 1). In the centre of the coprodeum the anal sphincter is visible, separating the coprodeum from the colon as a complete and moving structure (Fig. 1). Behind the edge of the anal sphincter, in the distal colon, urate depositions can frequently be seen (Fig. 1). When retracting the optic, several incomplete septa rising from the mucosa are visible. These membranes divide the chamber of the coprodeum into different sub-chambers. The urodeum in female vipers is characterized by three distinct chambers, which become visible immediately behind the exit of the sphincter of the coprodeum. The latter divides the urodeum from the coprodeum, and is visible in the upper part of the view (ventral in the image, being the animal in dorsal recumbency). The sphincter of the coprodeum is surrounded by a highly convoluted mucosa which contracts or relaxes, opening and closing the entrance of the coprodeum (Plate V, Fig. 2). In the lower (dorsal) part of the urodeum, two distinct chambers, the vaginal pouches, are visible. These structures lead to the entrance of the oviduct. The vaginal pouches terminate in a sphincter: the vaginal sphincter (Fig. 2). All structures of the urodeum of female vipers are divided by two highly developed septa. One septum divides the sphincter of the coprodeum from the vaginal pouches, and the other one divides the two vaginal pouches (Fig. 2). Aligning the optic with the vaginal pouches allows the exploration of the entrance of the oviducts. A cannula can easily be inserted into the caudal-most part of the oviduct overcoming the vaginal sphincter.

The urodeum of male vipers is characterized by two distinct structures. In the upper part of the view (ventral in the image, with the animal in dorsal recumbency), the sphincter of the coprodeum is visible. It is surrounded by a gently folded mucosa, far thinner in males compared to the females (Plate VI, Fig. 3). Looking downward, the urethral papillae are visible (dorsal in the image, with the animal in dorsal recumbency). These structures surface on a large mucosal protrusion emerging from the dorsal urodeum (Fig. 3). The urethral papillae are small structures and may be difficult to locate. However, urates are often visible leaking from the papillae, making their visualization easier (Fig. 3). The mucosal protrusion that hosts the urethral papillae is a shape-changing structure. It can be found in two different appearances: closed, (Fig. 3B) creating a distinct blind fold between the urethral papillae and the sphincter of the coprodeum; or open, (Fig. 3A) showing the open fold and the smooth mucosa between the urethral papillae and the sphincter of the coprodeum.

Finally, the optic can be almost completely withdrawn, revealing the proctodeum which is a tubular and muscular structure leading to the vent.

\section{Discussion}

Female vipers are characterized by the presence of relatively enlarged vaginas. These structures are referred to as vaginal pouches (Ludwing and Rahn 1943) and are a major characteristic of the female urodeum in Vipera ammodytes. In some non-viperid snakes the 
presence of a muscular sphincter has been described between the vaginal pouches and the oviduct by Uribe et al. (1998). The existence of a similar sphincter has been suggested in Vipera berus (Nilson andAndrén 1982). The function of the vaginal pouch, as suggested by Ludwing and Rahn (1943), is to aid copulation. According to these authors, the hemipenis of the male viper extends through the proctodeum of the female into the vaginal pouch during mating, to aid the sperm flow into the oviduct. The mechanics of viper copulation suggests that the vaginal sphincter, if closed and not properly stimulated by the mating, may hinder the entrance of sperm into the oviduct. Naturally, male vipers lack vaginal pouches and possess a mucosal protrusion in the centre of the urodeum. This structure may be involved in copulation, aiding the sperm flow from the genital papillae to the spermatic sulci of the hemipenis. In snakes, the opening of the ureter is separated from the opening of the sperm-ducts and oviducts (O'Malley 2005; Holtz 2006). However, our technique did not allow the differentiation of these small structures, except in males when urates exiting from the ureters were visible. Future studies should be conducted to determine the exact position of these structures. In Vipera ammodytes the presence of the vaginal pouches and the two septa dividing the urodeum in different chambers in the female, and the mucosal protrusion in the urodeum of the male viper, facilitates an accurate determination of the sex by cloacoscopy. We propose cloacoscopy as a method for sex determination in vipers when other more common techniques have failed to provide reliable results. The vaginal pouch of vipers may be explored and cannulated in order to deliver sperm into the oviduct (Plate VI, Fig. 4) during artificial insemination. The cannulation of the oviduct, overcoming the vaginal sphincter, may enhance the possibility of fertilization of the female viper. Cloacoscopy is a safe and effective method for the exploration of the cloaca of male and female vipers. It proved to be a useful tool for clinical examination and sex determination. This technique might be used to assist artificial insemination in Vipera ammodytes in the future.

\section{References}

Di Girolamo N, Selleri P 2015: Clinical Applications of Cystoscopy in Chelonians. Vet Clin North Am Exot Anim Pract 18: 507-526

Divers SJ 2014: Diagnostic endoscopy. In: Current therapy in reptile medicine and surgery. Mader DR, Divers SJ (Eds): Elsevier Saunders, St. Louis, pp 154-178

Holtz P 2006: Renal anatomy and physiology. In: Reptile medicine and surgery. Mader DR (Ed.) Elsevier Saunders, St. Louis, pp 135-144

Jekl V, Hauptman K, Knotek Z 2007: Cloacoscopy in chelonians - valuable diagnostic tool for reproductive tract evaluation. Proc. 43 rd. International Symposium on Diseases of Zoo and Wild Animals, Edinburgh $16^{\text {th }}-20^{\text {th }}$ May 2007, pp 162-163

Knotek Z, Jek1 V 2015: Pulmonoscopy of Snakes. Vet Clin North Am Exot Anim Pract 18: 493-506.

Ludwing M, Rahn H 1943: Sperm storage and copulatory adjustment in the prairie rattlesnake. Copeia 1: 15-28

Nilson G 1981: Ovarian cycle and reproductive dynamics in the female adder Vipera berus (Reptilia, Viperidae). Amphib-reptil 2: 62-84

O’Malley B 2005: Snakes. In: Clinical Anatomy and Physiology of Exotic Species. O’Malley B (Ed.) Elsevier Saunders, Philadelphia, pp 77-93

Stahl JS 2006: Introduction to cloacoscopy in snakes. In: Proc Abstracts The North American Veterinary Conference, 7-11 January 2006, 20: 1684-1685

Uribe MC, Gonzales-Portes G, Palmer BD, Giuliette LJ 1998: Cyclic histological changes of the oviductalcloacal junction in the viviparous snakes Toluca lineata. J Morphol 237: 91-100 
Plate V

Oliveri M. et al.: Cloacoscopy ....pp. 251-253

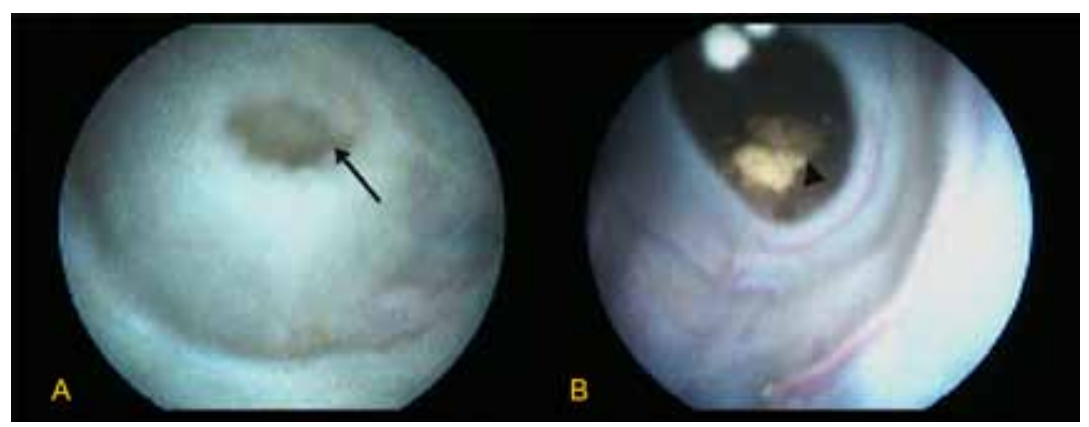

Fig. 1. A - Horned viper coprodeum (animal in dorsal recumbency). Smooth mucosa with moderate vasculature, free from urates. In the centre of the view, the anal sphincter is present (arrow) which separates the coprodeum from the colon. B - Behind the edge of the anal sphincter, deposition of urates (arrowhead) is seen in the distal colon.

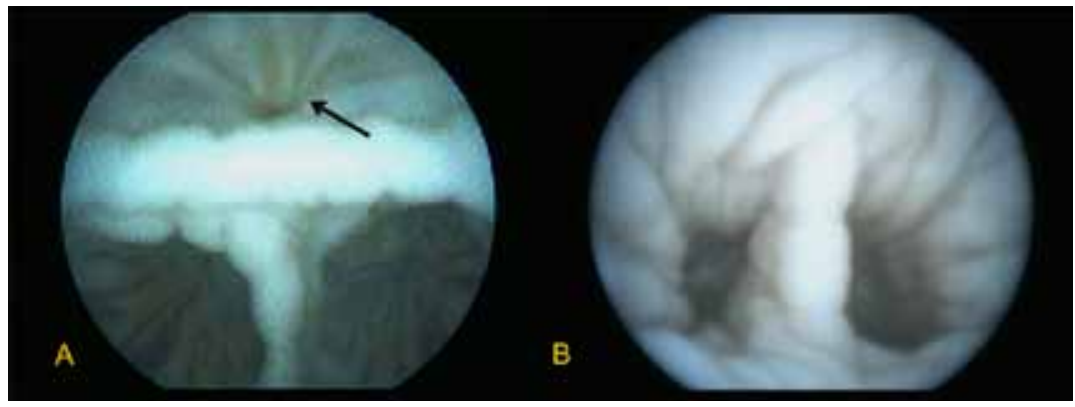

Fig. 2. A - Female horned viper urodeum (animal in dorsal recumbency). Three distinct chambers are visible: the entrance of the urodeum (arrow) and the two vaginal pouches. All structures of the urodeum are divided by two septa. One divides the sphincter of the coprodeum from the vaginal pouches (asterisk), and the second divides the two vaginal pouches (arrowhead). B - In the lower part of the view, two vaginal pouches are visible. The septum between the vaginal pouches is clearly visible (arrowhead). At the bottom of the two vaginal pouches, closed vaginal sphincters are visible. 


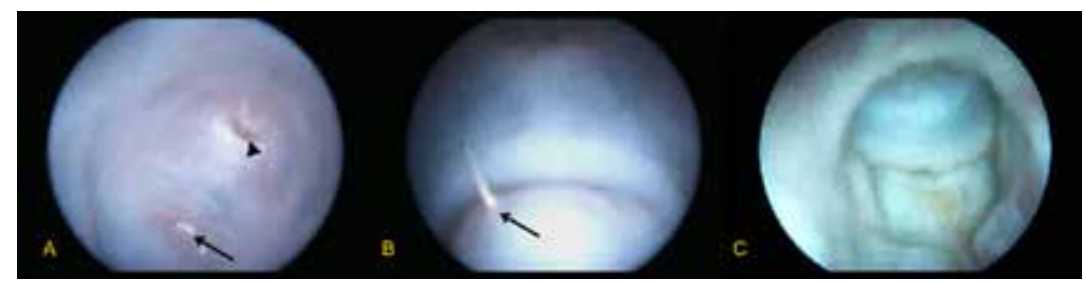

Fig. 3. Male horned viper urodeum (animal in dorsal recumbency). A - In the upper part of the view (ventral), the sphincter of the coprodeum is visible (arrowhead). The urinary papillae are visible as well (arrow), with urates surfacing from the ostium. B - Closed mucosal protrusion. $\mathrm{C}$ - Opened mucosal protrusion.

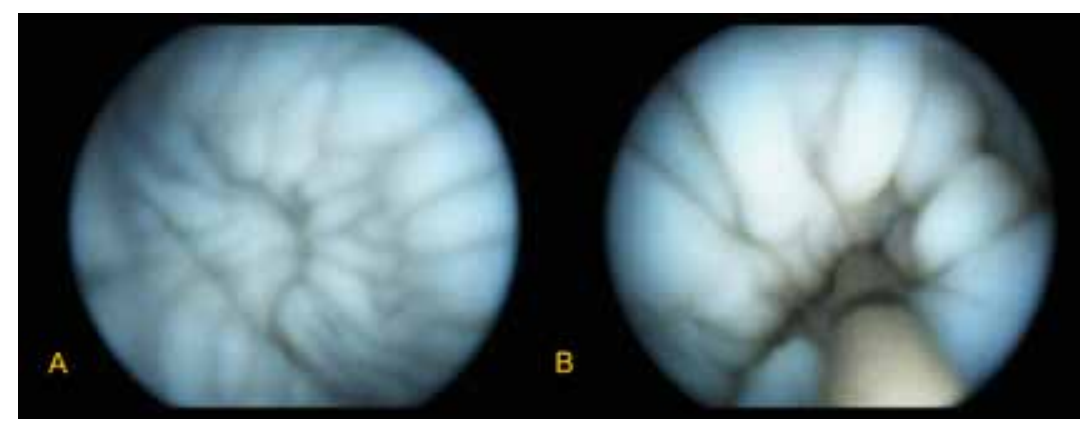

Fig. 4. Female horned viper (animal in dorsal recumbency). Examination of the vaginal pouch and cannulation of the oviduct. A - Convoluted mucosa and a tight sphincter in the bottom. B Insertion of a cannula into the oviduct. 